\title{
Micro-himenópteros associados com galhas de Cecidomyiidae (Diptera) em Restingas do Estado do Rio de Janeiro (Brasil)
}

\author{
Valeria Cid Maia $^{1,2}$ \& Maria Antonieta Pereira de Azevedo ${ }^{1}$ \\ ${ }^{1}$ Departamento de Entomologia, Museu Nacional, Quinta da Boa Vista, São Cristóvão, \\ CEP 20940-040, Rio de Janeiro, RJ, Brasil \\ ${ }^{2}$ Autor para correspondência: Valéria Cid Maia, e-mail: maiavcid@acd.ufrj.br
}

MAIA, V. C. \& AZEVEDO, M. A. P. Micro-Hymenoptera associated with Cecidomyiidae (Diptera) galls at Restingas of the Rio de Janeiro State. Biota Neotrop., 9(2): http://www.biotaneotropica.org.br/v9n2/en/ abstract?article+bn02809022009.

\begin{abstract}
Micro-Hymenoptera are very frequent on Cecidomyiidae (Diptera) galls and most important natural enemy of this Diptera. Although their ecological importance, little is known about the faunistic composition of this guild of parasitoids in Restinga areas. The collection of the Museu Nacional comprises a large number of micro-Hymenoptera collected during 16 years and identified at family level. It includes specimens of Aphelinidae, Bethylidae, Braconidae, Elasmidae, Encyrtidae, Eulophidae, Eupelmidae, Eurytomidae, Mymaridae, Platygastridae, Pteromalidae, Scelionidae, Tanaostigmatidae, Torymidae, and Signophoridae, totalizing 15 families. These microHymenoptera are associated with 45 plant species ( 35 genera and 25 families) and 26 Cecidomyiidae genera, being more frequent on Asphondylia, Bruggmannia, Clinodiplosis, Dasineura, Lopesia, Neolasioptera and Stephomyia galls. The great majority is parasitoid, but some of them, as Tanaostigmatidae and few species of Torymidae and Eulophidae, are inquilinous of galls (phytophagous). Eulophidae, Eupelmidae and Platygastridae were the most frequent families of Hymenoptera, being associated with the greatest number of families, genera and species of plant. Myrtaceae, Fabaceae and Malpighiaceae were the plant families with the greatest number of associated Hymenoptera families. About 135 new records of parasitoids on Cecidomyiidae galls are presented.
\end{abstract}

Keywords: Cecidomyiidae, Diptera, Hymenoptera, galls, Restingas, diversity.

MAIA, V. C. \& AZEVEDO, M. A. P. Micro-himenópteros associados com galhas de Cecidomyiidae (Diptera) em Restingas do Estado do Rio de Janeiro (Brasil). Biota Neotrop., 9(2): http://www.biotaneotropica.org.br/ v9n2/pt/abstract?article+bn02809022009.

Resumo: Os microhimenópteros são muito freqüentes em galhas de Cecidomyiidae (Diptera) e representam o principal inimigo natural dessa família de Diptera. Apesar de sua importância ecológica, pouco se conhece sobre a composição faunística dessa guilda de parasitóides em ambientes de Restinga. A coleção de micro-himenópteros do Museu Nacional abriga um grande número de exemplares coletados ao longo de 16 anos de coleta e identificados em nível de família. Inclui representantes de Aphelinidae, Bethylidae, Braconidae, Elasmidae, Encyrtidae, Eulophidae, Eupelmidae, Eurytomidae, Mymaridae, Platygastridae, Pteromalidae, Scelionidae, Tanaostigmatidae, Torymidae e Signophoridae, totalizando 15 famílias. Esses micro-himenópteros estão associados com 45 espécies de plantas hospedeiras ( 35 gêneros e 25 famílias) e 26 gêneros de Cecidomyiidae, sendo mais freqüentes em galhas de Asphondylia, Bruggmannia, Clinodiplosis, Dasineura, Lopesia, Neolasioptera e Stephomyia. A grande maioria é parasitóide, mas alguns representantes (Tanaostigmatidae e algumas espécies de Torymidae e Eulophidae) são fitófagos inquilinos de galhas. Eulophidae, Eupelmidae e Platygastridae destacaram-se como as famílias de Hymenoptera mais freqüentes, ocorrendo em um número maior de famílias, gêneros e espécies de plantas. Myrtaceae, Fabaceae e Malpighiaceae destacaram-se como as famílias de planta com maior número de famílias de Hymenoptera associadas. Cerca de 135 registros novos de parasitóides em galhas de Cecidomyiidae são apresentados.

Palavras-chave: Cecidomyiidae, Diptera, Hymenoptera, galhas, Restingas, diversidade. 


\section{Introdução}

Os micro-himenópteros são os mais importantes inimigos naturais dos Cecidomyiidae (Diptera) galhadores. São encontrados freqüentemente em galhas induzidas por esses mosquitos, atuando na maioria das vezes como parasitóides, e em alguns casos como fitófagos, capazes até de modificar a estrutura e a morfologia da galha. As espécies parasitóides podem ser desde generalistas a monófagas, gregárias ou isoladas, ecto ou endoparasitas.

Os micro-himenópteros parasitóides são representados por diversas famílias, especialmente por Platygastridae, Pteromalidae e Torymidae, menos comumente por Ceraphronidae, Encyrtidae, Eupelmidae e Eulophidae, e mais raramente por Braconidae e Ichneumonidae (Gagné 1989). Nas Restingas fluminenses, Maia 2001 apontou os Eulophidae, Eurytomidae e Platygastridae como as famílias mais freqüentes em galhas de Cecidomyiidae.

O conhecimento sobre os micro-himenópteros associados a galhas em ambientes de Restinga é escasso, principalmente no que se refere à taxonomia (devido à carência de especialistas no grupo e à escassez de levantamentos faunísticos) e à biologia das espécies. A primeira contribuição sobre micro-himenópteros parasitóides associados a galhas em Restingas deve-se a Maia \& Monteiro (1999). Esses autores investigaram as espécies cecidógenas e parasitóides associadas a Guapira opposita (Vell.) Reitz. (Nyctaginaceae) na Restinga da Barra de Maricá (Maricá, RJ), registrando 30 espécies de parasitóides. As espécies mais freqüentes foram classificadas em ecto ou endoparasitóides, gregárias ou isoladas e compuseram uma rede trófica, onde foi verificada a ocorrência de hiperparasitismo, multiparasitismo e poliembrionia.

No mesmo ano, Maia (1999) estudou a fauna de artrópodes associadas a duas galhas de Cecidomyiidae em duas famílias de plantas na Restinga da Barra de Maricá (Maricá, RJ), registrando a ocorrência de três espécies de micro-himenópteros parasitóides distribuídos em três famílias: Braconidae, Eurytomidae e Platygastridae.

Mais tarde, Maia \& Tavares (2000) estudaram a flutuação populacional de Cordiamyia globosa Maia, 1996 (Diptera, Cecidomyiidae), espécie galhadora, e os parasitóides associados a ela na Restinga da Barra de Maricá (Maricá, RJ), tendo registrado oito espécies parasitóides distribuídas em cinco famílias: Platygastridae, Pteromalidae, Torymidae, Eulophidae e Encyrtidae.

Posteriormente, Maia (2001) em um levantamento de galhas em Restingas fluminenses registrou a ocorrência de micro-himenópteros parasitóides e modificadores de galhas de 11 famílias diferentes em 64 morfotipos de galhas induzidas por Cecidomyiidae, o que equivale a $63 \%$ do total de morfotipos.

Maia \& Azevedo (2001a) publicaram informações sobre os Platygastridae associados a galhas em Restingas fluminenses, registrando a ocorrência de quatro gêneros parasitóides (cada qual representado por uma morfoespécie), associados a 15 espécies de Cecidomyiidae e 13 espécies de plantas.

Maia \& Azevedo (2001b) publicaram um segundo artigo na mesma linha sobre os Eurytomidae, registrando a ocorrência de 19 espécies parasitóides distribuídas em três gêneros e associadas a 17 espécies de Cecidomyiidae, (10 gêneros) e a 16 espécies de plantas (12 gêneros e 12 famílias).

Ferraz \& Monteiro (2003) estudaram as interações entre um Cecidomyiidae galhador, Myrciariamyia maricaensis Maia, 1995, e os micro-himenópteros associados a sua galha, tendo registrado duas espécies inquilinas, uma modificadora da galha e uma outra endogalhadora.

A maior parte dos registros tratam os micro-himenópteros em nível de família e ou gênero, o que demonstra o desconhecimento da fauna neotropical e a carência de taxonomistas no grupo.
As informações sobre os micro-himenópteros associados a galhas em Restingas estão pulverizadas na literatura; e desde 2003, nenhuma nova informação foi publicada.

A coleção de micro-himenópteros do Museu Nacional abriga um grande número de exemplares obtidos a partir da criação de galhas de Cecidomyiidae (Diptera) em Restingas do Estado do Rio de Janeiro. Refere-se a 16 anos de coleta e compreende muitos dados inéditos da relação planta hospedeira/inseto galhador/parasitóide. Esses dados são apresentados e discutidos no presente artigo, e visam contribuir para o conhecimento da diversidade dos Hymenoptera associados a galhas em Restingas fluminenses. Embora, refira-se a categorias taxonômicas supra-específicas, representa o primeiro esforço no sentido de reunir dados de literatura e dados de coleção para fornecer um panorama mais completo e consolidar o conhecimento da diversidade desses micro-himenópteros.

\section{Local de Estudo}

Áreas de Restinga de três municípios do Estado do Rio de Janeiro

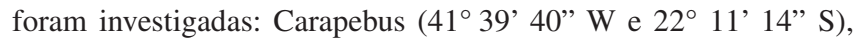
Maricá (42 49' 07" W e 22 $2^{\circ} 55^{\prime} 10^{\prime \prime} \mathrm{S}$, duas localidades: Barra de Maricá e Itaipuaçu) e Rio de Janeiro (Grumari: 43 31' 00" e $23^{\circ} 02$ ' 30” S).

Cada localidade foi investigada sistematicamente nos seguintes períodos: Carapebus: de maio, 1998 a abril, 1999; Barra de Maricá: desde 1992 até o presente; Itaipuaçu: de novembro, 1997 a outubro, 1998 e Grumari: de agosto, 2002 a julho, 2004.

As Restingas foram escolhidas como área de estudo por apresentarem grande riqueza de galhas e de parasitóides (Maia, 2001). São ambientes que se estendem por quase toda costa brasileira, cujas principais formações estão localizadas na Bahia, Espírito Santo, Rio de Janeiro e São Paulo.

Compreendem uma grande variedade de comunidades vegetais (Araújo \& Henriques, 1984) e diversas famílias de planta, com destaque para as Asteraceae (comuns nas comunidades psamófitas reptantes), Cyperaceae (nos brejos herbáceos), Myrtaceae (em "thickets"), Clusiaceae e Palmaceae (em "scrubs"), Bignoniaceae (nas florestas permanentemente inundadas), Bromeliaceae e Cactaceae (Araújo \& Henriques 1984).

Vários fatores foram relacionados positivamente à riqueza de galhas, entre eles estresse higrotérmico (Fernandes \& Price 1992). Devido aos contrastes diários de temperatura, umidade, rajadas de ventos, forte radiação e solo arenoso, as Restingas podem ser consideradas como ambientes com características de estresse higrotérmico (Monteiro et al. 2004), o que explicaria essa grande riqueza de galhas.

\section{Material e Métodos}

Os exemplares estudados encontram-se depositados na coleção de Hymenoptera do Museu Nacional/UFRJ (MNRJ), no Departamento de Entomologia. A grande maioria foi preservada em álcool $70 \%$ e parte dos representantes dos Eulopidae foi alfinetada após secagem em ponto crítico. Todo o material foi obtido por meio de criação, através do acondicionamento de galhas em potes plásticos forrados com papel umedecido, fechados com micro-tela e etiquetados. A maioria está guardada em micro-tubos contendo álcool 70\%, e uma pequena parte foi preservada a seco, estando montada em triângulos de cartolina.

Na etiqueta do material, além dos dados convencionais de coleta (local, data e coletor), constam informações sobre a espécie da planta hospedeira, morfotipo de galha e espécie galhadora.

Os espécimes estão organizados por família de Hymenoptera.e foram identificados por alguns especialistas, como John La Salle 
(CSIRO, Austrália), Celso Oliveira Azevedo (Universidade Federal do Espírito Santo), Marcelo Tavares (Universidade Federal do Espírito Santo) e Maria Angélica Penteado Dias (USP), mas principalmente por M.A.P. de Azevedo (Museu Nacional/UFRJ).

\section{Resultados e Discussão}

As Restingas estudadas totalizaram 106 morfotipos de galhas de Cecidomyiidae em 45 espécies de plantas (35 gêneros e 25 famílias). Micro-himenópteros foram obtidos de todas espécies de plantas galhadas e de $95 \%$ dos morfotipos de galhas. Esse valor é significantemente superior aos observados para áreas de Restinga em São Paulo e para áreas de cerrado em Minas Gerais (24\% e 34\%, respectivamente) (Maia, et al. 2008, Maia \& Fernandes 2004), indicando que frequiência de micro-himenópteros é bem mais alta nas Restingas fluminenses. No entanto, vale a pena ressaltar que as Restingas paulistas e o cerrado mineiro foram investigados por apenas 12 meses, enquanto que as Restingas fluminenses o foram por 16 anos, logo o tempo amostral foi muito diferenciado, resultando em um esforço de coleta igualmente distinto.

Os morfotipos de galhas para os quais não se registrou a presença de micro-himenópteros ocorreram em 10 espécies de plantas hospedeiras, cada qual pertencendo a uma família botânica distinta: Vernonia rufescens St. Hill (Asteraceae), Arrabidaea conjugata (Vell.) Mart. (Bignoniaceae), Selenicereus setaceus (SD) Berg. (Cactaceae), Jacquemontia holosericea (Weinmann) O' Donell (Convolvulaceae), Tetrapteris phlomoides (Spr.) Nied. (Malpighiaceae), Myrcia ovata Camb. (Myrtaceae), Passiflora mucronata Lam. (Passifloraceae), Piper divaricatum Meyer (Piperaceae), Paullinia weinmanniaefolia Mart. (Sapindaceae) e Smilax rufesxens Griseb (Smilacaceae).

Nas Restingas do Estado do Rio, as Asteraceae incluem duas espécies de plantas hospedeiras: Mikania hoehnei Robinson com dois morfotipos de galhas de Cecidomyiidae e $V$. rufescens com apenas um. Somente o morfotipo desta última planta não está associado com micro-himenópteros. A galha em $V$. rufescens é induzida por Asphondylia sp. Seis outras espécies desse gênero induzem galhas em plantas nas Restingas fluminenses, todas estão associadas a micro-himenópteros.

Arrabidaea conjugata é a única espécie de Bignoniaceae com registro de galha de Cecidomyiidae nas Restingas fluminenses. Três morfotipos são conhecidos, apenas um, induzido por um Cecidomyiinae não determinado, não tem associação com micro-himenópteros.

Dentre as Cactaceae, apenas S. setaceus apresenta galha de Cecidomyiidae. O indutor é Neolasioptera cerei Rübsaamen, 1905. Outras quatro espécies de Neolasioptera induzem galhas nessas Restingas, porém apenas a citada não possui associação com microhimenópteros.

J. holosericea é a única Convolvulaceae galhada nas Restingas fluminenses. Seu indutor é Schizomyia sp. Apenas mais uma espécie de Schizomyia Kieffer, 1889 induz galhas nas Restingas do Estado do Rio. Sua planta hospedeira é T. phlomoides (Malpighiaceae). Ambas espécies galhadoras não têm associação conhecida com micro-himenópteros. Outras duas Malpighiaceae possuem galhas de Cecidomyiidae estão associadas com micro-himenópteros.

Myrcia ovata é uma das oito espécies de Myrtaceae a apresentar galhas de Cecidomyiidae nas Restingas do Estado do Rio. Três morfotipos de galhas estão assinalados para essa planta. Apenas o morfotipo induzido por Dasineura sp. não tem micro-himenópteros associados. Outras quatro espécies desse gênero induzem galhas em outras plantas de Restinga, todas com associação com micro-himenópteros.

Passiflora mucronata e Piper divaricatum são as únicas Passifloraceae e Piperaceae com registro de galhas de Cecidomyiidae nas Restingas fluminenses. A galha em $P$. mucronata é induzida por Clinodiplosis sp. e a de $P$. divaricatum por um Cecidomyiinae não identificado. Outras 11 espécies de Clinodiplosis induzem galhas em Restingas, sendo que nove têm associação com microhimenópteros.

Paullinia weinmanniaefolia Mart. representa a única Sapindaceae das Restingas do Estado do Rio com galha de Cecidomyiidae. Três morfotipos estão assinalados, sendo que apenas o induzido por Clinodiplosis costai não está associado a micro-himenópteros.

Smilax rufescens é a única Smilacaceae com galha de Cecidomyiidae. Três morfotipos de galhas estão registrados, apenas um deles, induzido por Clinodiplosis sp. não tem associação conhecida com micro-himenópteros.

Os micro-himenópteros obtidos pertencem a 15 famílias: Aphelinidae, Bethylidae, Braconidae, Elasmidae, Encyrtidae, Eulophidae, Eupelmidae, Eurytomidae, Mymaridae, Platygastridae, Pteromalidae, Scelionidae, Tanaostigmatidae, Torymidae e Signophoridae.

A grande maioria atuou como parasitóide de galhadores, exceto Tanaostigmatidae e algumas espécies de Torymidae e Eulophidae, que atuaram como inquilinos de galhas (fitófagos).

Os Eulophidae, Eupelmidae e Platygastridae destacaram-se por ocorrer em um número maior de famílias, gêneros e espécies vegetais, seguidos pelos Eurytomidae, Torymidae e Pteromalidae (Tabela 1). Dentre eles, os Eulophidae representam a família de Chalcidoidea com maior riqueza de espécies. Diferentemente, os Eupelmidae, apesar de tão freqüentes em nosso estudo, são consideravelmente menos especiosos. Portanto, o número de registros dessas famílias de micro-himenópteros nas Restingas fluminenses não reflete a riqueza de espécies das mesmas.

Com relação às famílias de plantas, as Myrtaceae, Fabaceae e Malpighiaceae, apresentaram o maior número de famílias de Hymenoptera associadas (Tabela 1). As Myrtaceae compreendem cerca de três mil espécies no mundo e são mais diversificadas nas Américas e Austrália. Estão muito bem representadas nas Restingas fluminenses, contribuindo significativamente para a fisionomia desse ambiente. Constituem a família de planta hospedeira com maior riqueza de galhas de Cecidomyiidae em Restingas do Estado do Rio de Janeiro, com 25 morfotipos. Compõem, portanto, um complexo diversificado e abundante, capaz de suportar uma grande variedade de parasitóides.

As Fabaceae, uma das maiores famílias de planta dentre as dicotiledôneas, com cerca de 13 mil espécies no mundo, com distribuição predominantemente tropical e subtropical (Joly, 1977), não se destacam nas Restingas fluminenses pela riqueza de galhas de Cecidomyiidae, compreendendo apenas sete morfotipos (Maia, 2001; Oliveira \& Maia, 2005). No entanto, apresentaram uma fauna de micro-himenópteros associados bem diversificada.

As Malpighiaceae, família de planta com cerca de 1200 espécies distribuídas nos trópicos, especialmente na América do Sul (Joly, 1977), por sua vez, não se destacam nem pela riqueza de espécies, nem pela riqueza de galhas de Cecidomyiidae ( $\mathrm{n}=5$ morfotipos) nas Restingas fluminenses. E ainda assim, foram a terceira família de planta em riqueza de famílias de Hymenoptera.

Com base no exposto, podemos verificar que a diversidade dos parasitóides associados às galhas de Cecidomyiidae parece não estar relacionada nem com a riqueza de espécies da família de planta hospedeira e nem com a riqueza de galhas, e que seria necessário buscar outras explicações para os resultados encontrados, como por exemplo, os compostos químicos presentes no tecido galhado.O tanino pode ser citado como um desses compostos que dificulta o ataque de parasitóides. Curiosamente, a concentração de tanino nos tecidos mais externos de diversas galhas é maior do que nos tecidos sadios, criando uma proteção química favorável ao galhador (Price, 1984). 
Tabela 1. Distribuição dos micro-himenópteros por planta hospedeira, morfotipo de galha e Cecidomyiidae galhador nas restingas do Estado do Rio de Janeiro (Brasil).

Table 1. Distribution of micro-Hymenoptera per host plant, morphotype of gall and Cecidomyiidae galling at restingas of the State of Rio de Janeiro (Brazil).

\begin{tabular}{|c|c|c|c|c|}
\hline Famílias de Hymenoptera & $\begin{array}{c}\text { Famílias da planta } \\
\text { hospedeira }\end{array}$ & $\begin{array}{l}\text { Espécie da planta } \\
\text { hospedeira }\end{array}$ & Morfotipo da galha & $\begin{array}{c}\text { Cecidomyiidae } \\
\text { galhador }\end{array}$ \\
\hline Aphelinidae & Chrysobalanaceae & $\begin{array}{l}\text { Couepia ovalifolia } \\
\text { (Schott) Benth }\end{array}$ & foliar circular & $\begin{array}{l}\text { Dasineura couepiae } \\
\text { Maia, } 2001\end{array}$ \\
\hline Aphelinidae & Erythroxylaceae & $\begin{array}{l}\text { Erythroxylum } \\
\text { ovalifolium } \mathrm{Peyr}\end{array}$ & botão floral & Asphondylia sp.1 \\
\hline Aphelinidae & Myrtaceae & $\begin{array}{l}\text { Myrciaria floribunda } \\
\text { (West ex Willd.) Berg }\end{array}$ & $\begin{array}{l}\text { enrolamento da } \\
\text { margem foliar }\end{array}$ & $\begin{array}{l}\text { Dasineura myrciariae } \\
\text { Maia, } 1996\end{array}$ \\
\hline Aphelinidae & Sapotaceae & $\begin{array}{l}\text { Manilkara subsericea } \\
\text { (Mart.) Dubard }\end{array}$ & foliar circular & Cecidomyiidi \\
\hline Aphelinidae: Encarsia sp. & Clusiaceae & $\begin{array}{l}\text { Clusia lanceolata } \\
\text { Camb. }\end{array}$ & foliar circular & $\begin{array}{l}\text { Clusiamyia nitida } \\
\text { Maia, } 1997\end{array}$ \\
\hline Bethylidae: Goniozus sp. & Burseraceae & $\begin{array}{l}\text { Protium brasiliense } \\
\text { (Spr.) Engl. }\end{array}$ & $\begin{array}{l}\text { enrolamento da } \\
\text { margem foliar }\end{array}$ & Lopesia sp.1 \\
\hline Bethylidae. & Myrtaceae & M. floribunda & $\begin{array}{l}\text { enrolamento da } \\
\text { margem foliar* }\end{array}$ & D. myrciariae \\
\hline Braconidae & Chrysobalanaceae & C. ovalifolia & foliar circular* & D. couepiae \\
\hline Braconidae & Fabaceae & Inga laurina (Sw.) Willd. & espess. nervura & Neolasioptera sp.1 \\
\hline Braconidae & Fabaceae & Inga maritima Benth. & espess.nervura* & Neolasioptera $\mathrm{sp} .2$ \\
\hline $\begin{array}{l}\text { Braconidae: } \\
\text { Donquickeia sp. }\end{array}$ & Myrtaceae & $\begin{array}{l}\text { Eugenia rotundifolia } \\
\text { Casar }\end{array}$ & cilíndrica caulinar & $\begin{array}{l}\text { Stephomyia } \\
\text { rotundifoliorum Maia, } 1994\end{array}$ \\
\hline Braconidae & Myrtaceae & E. multiflora Camb. & digitiforme* & S. rotundifoliorum \\
\hline Braconidae & Myrtaceae & $\begin{array}{l}\text { Neomitranthes obscura } \\
\text { (DC) N. J. E. Silveira }\end{array}$ & cônica foliar* & $\begin{array}{l}\text { Stephomyia mina } \\
\text { Maia, } 1994\end{array}$ \\
\hline Braconidae & Sapindaceae & $\begin{array}{l}\text { Paullinia } \\
\text { weinmanniaefolia Mart. }\end{array}$ & cônica foliar* & $\begin{array}{l}\text { Palliniamyia ampla } \\
\text { Maia, } 2001\end{array}$ \\
\hline Eulophidae & Asclepiadaceae & $\begin{array}{l}\text { Peplonia asteria (Vell) } \\
\text { Font \& Schw }\end{array}$ & botão floral & $\begin{array}{l}\text { Asphondylia peploniae } \\
\text { Maia, } 2001\end{array}$ \\
\hline Eulophidae & Asteraceae & $\begin{array}{l}\text { Mikania hoehnei } \\
\text { Robinson }\end{array}$ & enrolamento foliar & Clinodiplosis sp.1 \\
\hline $\begin{array}{l}\text { Eulophidae: } \\
\text { Galeopsomyia } \mathrm{sp} .1 \\
\text { Cirrospilus } \text { sp.1 }\end{array}$ & Boraginaceae & Cordia verbenacea DC. & globosa foliar & $\begin{array}{l}\text { Cordiamyia globosa } \\
\text { Maia, } 1996\end{array}$ \\
\hline $\begin{array}{l}\text { Eulophidae: } \\
\text { Aprostocetus sp. }\end{array}$ & Burseraceae & P. brasiliense & $\begin{array}{l}\text { enrolamento da } \\
\text { margem foliar }\end{array}$ & Lopesia sp.1 \\
\hline Eulophidae & Burseraceae & P.brasiliense & cônica foliar* & Cecidomyiidi \\
\hline Eulophidae & Burseraceae & P. brasiliense & cônica curva* & Cecidomyiinae \\
\hline $\begin{array}{l}\text { Eulophidae: Xanthellum sp.1 } \\
\text { Tetrastichinae (gen.nov.) }\end{array}$ & Celastraceae & $\begin{array}{l}\text { Maytenus ovalifolia } \\
\text { Mart. }\end{array}$ & foliar circular & $\begin{array}{l}\text { Mayteniella distincta } \\
\text { Maia, } 2001\end{array}$ \\
\hline Eulophidae & Celastraceae & M. ovalifolia & fruto* & $\begin{array}{l}\text { Bruggmanniella } \\
\text { maytenuse } \\
\text { (Maia \& Couri, 1992) }\end{array}$ \\
\hline Eulophidae & Chrysobalanaceae & C. ovalifolia & foliar circular* & D. couepiae \\
\hline Eulophidae & Clusiaceae & C. lanceolata & esferóide foliar* & C. nitida \\
\hline $\begin{array}{l}\text { Eulophidae: } \\
\text { gen.nov.6sp.nov.1 }\end{array}$ & Erythroxylaceae & E. ovalifolium & triangular foliar & Lasiopteridi \\
\hline Eulophidae & Erythroxylaceae & E. ovalifolium & botão floral* & Asphondylia sp.1 \\
\hline $\begin{array}{l}\text { Eulophidae : } \\
\text { gen.nov.6sp.nov.3 }\end{array}$ & Erythroxylaceae & E. ovalifolium & cônica (gema)* & Cecidomyiinae \\
\hline Eulophidae & Euphorbiaceae & $\begin{array}{l}\text { Sebastiania glandulosa } \\
\text { (Mart.) Pax. }\end{array}$ & esférica (gema)* & $\begin{array}{l}\text { Schizomyia spherica } \\
\text { Maia \& Oliveira } 2007\end{array}$ \\
\hline Eulophidae & Euphorbiaceae & S. glandulosa & cônica (gema)* & $\begin{array}{l}\text { Clinodiplosis conica } \\
\text { Oliveira \& Maia, } 2008\end{array}$ \\
\hline
\end{tabular}


Tabela 1. Continuação...

\begin{tabular}{|c|c|c|c|c|}
\hline Famílias de Hymenoptera & $\begin{array}{c}\text { Famílias da planta } \\
\text { hospedeira }\end{array}$ & $\begin{array}{c}\text { Espécie da planta } \\
\text { hospedeira }\end{array}$ & Morfotipo da galha & $\begin{array}{c}\text { Cecidomyiidae } \\
\text { galhador }\end{array}$ \\
\hline $\begin{array}{l}\text { Eulophidae: } \\
\text { Aprostocetus sp.1 }\end{array}$ & Euphorbiaceae & S. glandulosa & $\begin{array}{l}\text { enrolamento da } \\
\text { margem foliar* }\end{array}$ & Cecidomyiidi \\
\hline $\begin{array}{l}\text { Eulophidae: } \\
\text { Chrysonotomyia sp. }\end{array}$ & Fabaceae & $\begin{array}{l}\text { Andira fraxinifolia } \\
\text { Benth. }\end{array}$ & circular foliar* & Meunieriella sp.1 \\
\hline Elasmus sp. & Fabaceae & I. laurina & espess. nervura & Neolasioptera sp.1 \\
\hline Eulophidae & Fabaceae & I. laurina & espess. nervura & Neolasioptera sp.1 \\
\hline $\begin{array}{l}\text { Eulophidae: } \\
\text { Xanthellum sp. } 1\end{array}$ & Fabaceae & I. laurina & fruto & Cecidomyiinae \\
\hline Eulophidae & Fabaceae & I. laurina & tubular foliar* & Cecidomyiinae \\
\hline Eulophidae & Fabaceae & I. maritima & espess.nervura* & Neolasioptera $\mathrm{sp} .2$ \\
\hline $\begin{array}{l}\text { Eulophidae: } \\
\text { Aprostocetus sp.1 }\end{array}$ & Loranthaceae & $\begin{array}{l}\text { Psittacanthus dichrous } \\
\text { (Mart.) Mart. }\end{array}$ & circular foliar * & $\begin{array}{l}\text { Costadiplosis maricaensis } \\
\text { Viceconte \& Maia, prelo }\end{array}$ \\
\hline $\begin{array}{l}\text { Eulophidae: } \\
\text { gen.nov.7sp.nov.1 }\end{array}$ & Malpighiaceae & Byrsonima sericea DC. & espess.caulinar* & Cecidomyiinae \\
\hline Eulophidae & Malpighiaceae & B. sericea & circular foliar & Oligotrohini \\
\hline $\begin{array}{l}\text { Eulophidae: } \\
\text { Aprostocetus sp. } 1\end{array}$ & Malpighiaceae & Heteropteris nitida DC. & botão floral* & $\begin{array}{l}\text { Clinodiplosis floricola } \\
\text { Novo-Guedes \& } \\
\text { Maia, } 2008\end{array}$ \\
\hline $\begin{array}{l}\text { Eulophidae: } \\
\text { Aprostocetus sp.1 } \\
\text { Pentastichus sp. } 2\end{array}$ & Myrsinaceae & $\begin{array}{l}\text { Rapanea parvifolia } \\
\text { (A. DC.) Mez. }\end{array}$ & circular foliar* & Cecidomyiinae \\
\hline $\begin{array}{l}\text { Eulophidae: } \\
\text { Quadrastichus sp.1 } \\
\text { gen.nov.4 sp.1 } \\
\text { Aprostocetus sp.1 }\end{array}$ & Myrtaceae & $\begin{array}{l}\text { Eugenia } \\
\text { copacabanensis } \\
\text { Kiaersk. }\end{array}$ & fusiforme foliar & $\begin{array}{l}\text { Stephomyia teralobae } \\
\text { Maia, } 1994\end{array}$ \\
\hline $\begin{array}{l}\text { Eulophidae: Tetrastichinae } \\
\text { (gen. nov.) }\end{array}$ & Myrtaceae & E. copacabanensis & espiralada foliar & $\begin{array}{l}\text { Stephomyia espiralis } \\
\text { Maia, } 1994\end{array}$ \\
\hline Eulophidae & Myrtaceae & E. multiflora & $\begin{array}{l}\text { enrolamento da } \\
\text { margem foliar }\end{array}$ & Cecidomyiinae \\
\hline Eulophidae & Myrtaceae & E. multiflora & piriforme foliar & Cecidomyiinae \\
\hline Eulophidae & Myrtaceae & E. multiflora & claviforme & $\begin{array}{l}\text { Stephomyia clavata } \\
\text { Tavares, } 1920\end{array}$ \\
\hline $\begin{array}{l}\text { Eulophidae: } \\
\text { Aprostocetus sp. } 2\end{array}$ & Myrtaceae & E. ovalifolia Camb. & espess.caulinar* & Não determinado \\
\hline $\begin{array}{l}\text { Eulophidae: } \\
\text { Closterocerus sp.1 } \\
\text { Pentastichus sp.1 } \\
\text { gen.nov.6 sp.nov. } 2 \\
\text { gen.nov.4 sp.nov.1 }\end{array}$ & Myrtaceae & E. rotundifolia & $\begin{array}{l}\text { enrolamento da } \\
\text { margem foliar }\end{array}$ & Cecidomyiidi \\
\hline Eulophidae & Myrtaceae & E. rotundifolia & circular foliar* & $\begin{array}{l}\text { Dasineura globosa } \\
\text { Maia, } 1996\end{array}$ \\
\hline Eulophidae & Myrtaceae & E. rotundifolia & cilíndrica caulinar* & S. rotundifoliorum \\
\hline $\begin{array}{l}\text { Eulophidae: } \\
\text { Chrysonotomyia sp. } \\
\text { gen.nov.2sp.nov.1 } \\
\text { Aprostocetus sp.1 }\end{array}$ & Myrtaceae & E. uniflora $\mathrm{L}$. & cônica foliar & $\begin{array}{l}\text { Clinodiplosis profusa } \\
\text { Maia, } 2001\end{array}$ \\
\hline Eulophidae & Myrtaceae & E. uniflora & triangular foliar* & Cecidomyiinae \\
\hline Eulophidae & Myrtaceae & E. uniflora & circular foliar & $\begin{array}{l}\text { Neolasioptera eugeniae } \\
\text { Maia, } 1993\end{array}$ \\
\hline $\begin{array}{l}\text { Eulophidae: } \\
\text { gen.nov.1sp.nov.1 } \\
\text { gen.nov.3sp.nov.1 } \\
\text { Aprostocetus } \text { sp.3 }\end{array}$ & Myrtaceae & M. ovata & gema & $\begin{array}{l}\text { Myrciamyia maricensis } \\
\text { Maia, } 1996\end{array}$ \\
\hline
\end{tabular}


Tabela 1. Continuação...

\begin{tabular}{|c|c|c|c|c|}
\hline Famílias de Hymenoptera & $\begin{array}{c}\text { Famílias da planta } \\
\text { hospedeira }\end{array}$ & $\begin{array}{c}\text { Espécie da planta } \\
\text { hospedeira }\end{array}$ & Morfotipo da galha & $\begin{array}{c}\text { Cecidomyiidae } \\
\text { galhador }\end{array}$ \\
\hline Eulophidae: Tetrastichinae & Myrtaceae & M. ovata & globular foliar & Dasineura sp.1 \\
\hline Eulophidae: Proacrias sp. & Myrtaceae & M. floribunda & $\begin{array}{l}\text { enrolamento da } \\
\text { margem foliar }\end{array}$ & D. myrciariae \\
\hline $\begin{array}{l}\text { Eulophidae: } \\
\text { gen.nov.3sp.nov. }\end{array}$ & Myrtaceae & M. floribunda & bivalva (gema)* & $\begin{array}{l}\text { Myrciariamyia bivalva } \\
\text { Maia, } 1995\end{array}$ \\
\hline $\begin{array}{l}\text { Eulophidae: } \\
\text { Quadrastichus sp. } \\
\text { Aprostocetus sp.4 }\end{array}$ & Myrtaceae & N. obscura & cônica foliar & S. mina \\
\hline $\begin{array}{l}\text { Eulophidae: } \\
\text { Quadrastichus sp. } \\
\text { Tetrastichinae (gen.nov.) }\end{array}$ & Myrtaceae & N. obscura & imbricada (gema)* & $\begin{array}{l}\text { Neomitranthella robusta } \\
\text { Maia, } 1996\end{array}$ \\
\hline $\begin{array}{l}\text { Eulophidae: } \\
\text { Aprostocetus sp.1 }\end{array}$ & Myrtaceae & N. obscura & triangular foliar* & Cecidomyiinae \\
\hline $\begin{array}{l}\text { Eulophidae: } \\
\text { Galeopsomyia sp.1 } \\
\text { Chrysotomyia } \mathrm{sp} .\end{array}$ & Nyctaginaceae & $\begin{array}{l}\text { Guapira opposita (Vell.) } \\
\text { Reitz. }\end{array}$ & globosa foliar & $\begin{array}{l}\text { Bruggmannia robusta } \\
\text { Maia \& Couri, } 1993\end{array}$ \\
\hline $\begin{array}{l}\text { Eulophidae: } \\
\text { Galeopsomyia sp.1 } \\
\text { Chrysotomyia } \mathrm{sp} . \\
\text { Cirrospilus } \mathrm{sp} .\end{array}$ & Nyctaginaceae & G. opposita & circular foliar & $\begin{array}{l}\text { Bruggmannia elongata } \\
\text { Maia \& Couri, } 1993\end{array}$ \\
\hline $\begin{array}{l}\text { Eulophidae: } \\
\text { Galeopsomyia sp.1 }\end{array}$ & Nyctaginaceae & G. opposita & triangular foliar & $\begin{array}{l}\text { Bruggmannia acaudata } \\
\text { Maia, } 2004\end{array}$ \\
\hline Eulophidae & Nyctaginaceae & G. opposita & foliar roxa* & Bruggmannia sp.1 \\
\hline $\begin{array}{l}\text { Eulophidae: } \\
\text { Aprostocetus sp.5 }\end{array}$ & Ochnaceae & $\begin{array}{l}\text { Ouratea cuspidata } \\
\text { (St. Hil.) Engl. }\end{array}$ & barril foliar* & Contarinia sp. \\
\hline Eulophidae: Horismenus sp. & Rubiaceae & $\begin{array}{l}\text { Borreria verticillata (L.) } \\
\text { Meyer }\end{array}$ & inflorescência & $\begin{array}{l}\text { Asphondylia borreriae } \\
\text { Rübsaamen, } 1905\end{array}$ \\
\hline $\begin{array}{l}\text { Eulophidae } \\
\text { gen.nov. } 2 \text { sp.nov. } 2 \\
\text { gen.nov. } 5 \text { sp.nov.1 } \\
\text { Aprostocetus sp.1 }\end{array}$ & Sapindaceae & P.weinmanniaefolia & cônica foliar* & P. ampla \\
\hline Eulophidae: Miotropis sp. & Solanaceae & Solanum affine Sendth & globular foliar & Cecidomyiinae \\
\hline $\begin{array}{l}\text { Eulophidae: } \\
\text { Hadrotrichodes sp. } \\
\text { Closterocerus sp. }\end{array}$ & Sapotaceae & M. subsericea & foliar circular & Cecidomyiidi \\
\hline Eulophidae: Xanthobium sp. & Sapotaceae & $\begin{array}{l}\text { Pouteria caimito } \\
\text { (R. \& P.) Radlk. }\end{array}$ & tubular foliar* & $\begin{array}{l}\text { Youngomyia pouteriae } \\
\text { Maia, } 2001\end{array}$ \\
\hline $\begin{array}{l}\text { Eulophidae: Tetrastichinae } \\
\text { (gen.nov.) }\end{array}$ & Sapotaceae & $\begin{array}{l}\text { Pouteria venosa (Mart.) } \\
\text { Baehni }\end{array}$ & circular foliar & $\begin{array}{l}\text { Lopesia singularis Maia, } \\
\text { Maia, } 2001\end{array}$ \\
\hline $\begin{array}{l}\text { Eulophidae: } \\
\text { Pentastichus sp.3 }\end{array}$ & Smilacaceae & Smilax rufescens Griseb & circular foliar & $\begin{array}{l}\text { Smilasioptera candelariae } \\
\text { Möhn, } 1975\end{array}$ \\
\hline Eulophidae & Verbenaceae & Lantana camara $\mathrm{L}$. & globosa foliar* & $\begin{array}{l}\text { Schismatodiplosis lantanae } \\
\text { Rübsaamen, } 1916\end{array}$ \\
\hline Encyrtidae & Fabaceae & I. maritima & espess. nervura & Neolasioptera sp.2 \\
\hline Encyrtidae & Malpighiaceae & B. sericea & botão floral & $\begin{array}{l}\text { Bruggmanniella } \\
\text { byrsonimae } \\
\text { (Maia \& Couri, 1992) }\end{array}$ \\
\hline Encyrtidae & Myrtaceae & M. floribunda & $\begin{array}{l}\text { enrolamento da } \\
\text { margem foliar }\end{array}$ & D. myrciariae \\
\hline Encyrtidae & Myrtaceae & M. floribunda & bivalva (gema) & M. bivalva \\
\hline Encyrtidae & Myrtaceae & N. obscura & $\begin{array}{l}\text { enrolamento da } \\
\text { margem foliar }\end{array}$ & $\begin{array}{l}\text { Dasineura tavaresi } \\
\text { Maia, } 1996\end{array}$ \\
\hline Encyrtidae & Olacaceae & Ximenia americana $\mathrm{L}$. & espess. caulinar & $\begin{array}{l}\text { Asphondylia communis } \\
\text { Maia \& Couri, } 1992\end{array}$ \\
\hline
\end{tabular}


Tabela 1. Continuação...

\begin{tabular}{|c|c|c|c|c|}
\hline Famílias de Hymenoptera & $\begin{array}{c}\text { Famílias da planta } \\
\text { hospedeira }\end{array}$ & $\begin{array}{c}\text { Espécie da planta } \\
\text { hospedeira }\end{array}$ & Morfotipo da galha & $\begin{array}{c}\text { Cecidomyiidae } \\
\text { galhador }\end{array}$ \\
\hline Encyrtidae & Sapotaceae & M. subsericea & foliar circular & Cecidomyiidi \\
\hline Eupelmidae & Boraginaceae & C. verbenacea & espess.nervura* & Lopesiini \\
\hline $\begin{array}{l}\text { Eupelmidae: } \\
\text { Brasema sp. }\end{array}$ & Burseraceae & P. brasiliense & $\begin{array}{l}\text { enrolamento da } \\
\text { margem foliar }\end{array}$ & Lopesia sp.1 \\
\hline Eupelmidae & Burseraceae & P. brasiliense & cônica curva* & Cecidomyiinae \\
\hline Eupelmidae & Burseraceae & $\begin{array}{l}\text { P. heptaphyllum } \\
\text { (Aublet.) March. }\end{array}$ & gotiforme & Cecidomyiidi \\
\hline Eupelmidae & Burseraceae & P. heptaphyllum & $\begin{array}{l}\text { enrolamento da } \\
\text { margem foliar }\end{array}$ & Cecidomyiidi \\
\hline Eupelmidae & Celastraceae & M. ovalifolia & foliar circular* & M. distincta \\
\hline Eupelmidae & Chrysobalanaceae & C. ovalifolia & foliar circular* & D. couepiae \\
\hline Eupelmidae & Chrysobalanaceae & C. ovalifolia & $\begin{array}{l}\text { enrolamento da } \\
\text { margem foliar }\end{array}$ & $\begin{array}{l}\text { Lopesia marginalis } \\
\text { Maia, } 2001\end{array}$ \\
\hline Eupelmidae & Clusiaceae & $\begin{array}{l}\text { Clusia hilariana } \\
\text { Schltdl. }\end{array}$ & esferóide foliar & $\begin{array}{l}\text { Clusiamyia granulosa } \\
\text { Maia, } 2001\end{array}$ \\
\hline Eupelmidae & Clusiaceae & C. lanceolata & esferóide foliar* & C. nitida \\
\hline Eupelmidae & Erythroxylaceae & E. ovalifolium & triangular foliar* & Lasiopteridi \\
\hline Eupelmidae & Erythroxylaceae & E. ovalifolium & cônica (gema) & Cecidomyiinae \\
\hline Eupelmidae & Euphorbiaceae & S. glandulosa & $\begin{array}{l}\text { enrolamento da } \\
\text { margem foliar }\end{array}$ & Cecidomyiidi \\
\hline Eupelmidae: Brasema sp. & Fabaceae & I. laurina & espess. nervura & Neolasioptera sp.1 \\
\hline Eupelmidae & Fabaceae & I. laurina & tubular foliar* & Cecidomyiinae \\
\hline Eupelmidae & Fabaceae & I. maritima & espess.nervura* & Neolasioptera sp.2 \\
\hline Eupelmidae & Malpighiaceae & B. sericea & espess.caulinar* & Cecidomyiinae \\
\hline Eupelmidae & Malpighiaceae & B. sericea & inflorescência & B. byrsonimae \\
\hline Eupelmidae & Myrtaceae & E. copacabanensis & cônica & S. tetralobae \\
\hline Eupelmidae & Myrtaceae & E. multiflora & claviforme & S. clavata \\
\hline Eupelmidae & Myrtaceae & E. multiflora & digitiforme & S.rotundifoliorum \\
\hline Eupelmidae & Myrtaceae & E. multiflora & piriforme foliar & Cecidomyiinae \\
\hline $\begin{array}{l}\text { Eupelmidae: } \\
\text { Brasema sp. }\end{array}$ & Myrtaceae & E. rotundifolia & cilíndrica caulinar* & S.rotundifoliorum \\
\hline Eupelmidae & Myrtaceae & E. uniflora & cônica foliar* & C. profusa \\
\hline Eupelmidae & Myrtaceae & E. uniflora & triangular no fruto & Cecidomyiinae \\
\hline Eupelmidae & Myrtaceae Myrtaceae & M. ovata & globular foliar* & Dasineura sp.1 \\
\hline Eupelmidae & Myrtaceae & M. floribunda & $\begin{array}{l}\text { enrolamento da } \\
\text { margem foliar* }\end{array}$ & D. myrciariae \\
\hline Eupelmidae & Myrtaceae & N. obscura & cônica foliar* & S. mina \\
\hline Eupelmidae & Myrtaceae & N. obscura & triangular foliar* & Cecidomyiinae \\
\hline Eupelmidae & Myrtaceae & N. obscura & imbricada (gema) & N. robusta \\
\hline Eupelmidae & Nyctaginaceae & G. opposita & globosa foliar* & B. robusta \\
\hline Eupelmidae & Nyctaginaceae & G. opposita & circular foliar & B. elongata \\
\hline Eupelmidae & Nyctaginaceae & G. opposita & triangular foliar* & B. acaudata \\
\hline Eupelmidae & Nyctaginaceae & G. opposita & espess.caulinar* & $\begin{array}{l}\text { Proasphondylia guapirae } \\
\text { Maia, } 1994\end{array}$ \\
\hline Eupelmidae & Ochnaceae & O. cuspidata & barril foliar* & Contarinia sp.1 \\
\hline Eupelmidae & Olacaceae & X. americana & espess.caulinar* & A. communis \\
\hline Eupelmidae & Rubiaceae & B. verticillata & inflorescência* & A. borreriae \\
\hline Eupelmidae & Sapindaceae & P.weinmanniaefolia & cônica foliar & P. ampla \\
\hline Eupelmidae & Sapotaceae & M. subsericea & foliar circular* & Cecidomyiidi \\
\hline Eupelmidae & Sapotaceae & P. caimito & tubular foliar & Y. pouteriae \\
\hline Eupelmidae & Sapotaceae & P. venosa & circular foliar & L. singularis \\
\hline
\end{tabular}


Tabela 1. Continuação...

\begin{tabular}{|c|c|c|c|c|}
\hline Famílias de Hymenoptera & $\begin{array}{c}\text { Famílias da planta } \\
\text { hospedeira }\end{array}$ & $\begin{array}{c}\text { Espécie da planta } \\
\text { hospedeira }\end{array}$ & Morfotipo da galha & $\begin{array}{c}\text { Cecidomyiidae } \\
\text { galhador }\end{array}$ \\
\hline Eupelmidae & Smilacaceae & S. rufescens & circular foliar* & S. candelariae \\
\hline $\begin{array}{l}\text { Eurytomidae: } \\
\text { Rileya } \mathrm{sp} .1\end{array}$ & Asclepiadaceae & P. asteria & botão floral & A. peploniae \\
\hline $\begin{array}{l}\text { Eurytomidae: } \\
\text { Eurytoma } \mathrm{sp} 1 .\end{array}$ & Bignoniaceae & $\begin{array}{l}\text { Arrabidaea conjugata } \\
\text { (Vell.) Mart. }\end{array}$ & cônica foliar & $\begin{array}{l}\text { Arrabidaeamyia serrata } \\
\text { Maia, } 2001\end{array}$ \\
\hline $\begin{array}{l}\text { Eurytomidae: } \\
\text { Eurytoma sp.2. }\end{array}$ & Boraginaceae & C. verbenacea & botão floral & $\begin{array}{l}\text { Asphondylia cordiae } \\
\text { Möhn, } 1959\end{array}$ \\
\hline $\begin{array}{l}\text { Eurytomidae: } \\
\text { Eurytoma sp.3 }\end{array}$ & Boraginaceae & C. verbenacea & globosa foliar & C. globosa \\
\hline Eurytomidae: & Burseraceae & P. brasiliense & cônica curva & Cecidomyiinae \\
\hline $\begin{array}{l}\text { Eurytomidae: } \\
\text { Rileya sp. } 2\end{array}$ & Clusiaceae & $\begin{array}{l}\text { Clusia fluminensis } \\
\text { Tr. \& Pl. }\end{array}$ & circular foliar & $\begin{array}{l}\text { Parazalepidota clusiae } \\
\text { Maia, } 2001\end{array}$ \\
\hline $\begin{array}{l}\text { Eurytomidae: } \\
\text { Eurytoma sp. } 4\end{array}$ & Clusiaceae & C. hilariana & circular foliar & C. granulosa Maia, 2001 \\
\hline $\begin{array}{l}\text { Eurytomidae: } \\
\text { Eurytoma sp.5 }\end{array}$ & Clusiaceae & C. lanceolata & esferóide foliar & C. nitida \\
\hline Eurytomidae & Erythroxylaceae & E. ovalifolium & botão floral & Asphondylia sp.1 \\
\hline $\begin{array}{l}\text { Eurytomidae: } \\
\text { Eurytoma sp.6}\end{array}$ & Erythroxylaceae & E. ovalifolium & cônica (gema) & Cecidomyiinae \\
\hline $\begin{array}{l}\text { Eurytomidae: } \\
\text { Rileya } \mathrm{sp} .3\end{array}$ & Euphorbiaceae & S. glandulosa & esférica (gema) & S. spherica \\
\hline Eurytomidae: Eurytoma sp. & Fabaceae & I. laurina & tubular foliar* & Meunieriella sp. \\
\hline Eurytomidae & Loranthaceae & $\begin{array}{l}\text { Struthanthus maricensis } \\
\text { Rizz. }\end{array}$ & ovóide na nervura* & $\begin{array}{l}\text { Asphondylia maricensis } \\
\text { Maia \& Couri, } 1992\end{array}$ \\
\hline $\begin{array}{l}\text { Eurytomidae: Sycophyla sp. } \\
\text { Eurytoma sp. }\end{array}$ & Malpighiaceae & B. sericea & espess.caulinar & Cecidomyiinae \\
\hline Eurytomidae & Malpighiaceae & B. sericea & botão floral & B. byrsonimae \\
\hline $\begin{array}{l}\text { Eurytomidae: } \\
\text { Rileya } \text { sp. } 6\end{array}$ & Myrtaceae & E. copacabanensis & fusiforme foliar & S. tetralobae \\
\hline $\begin{array}{l}\text { Eurytomidae: } \\
\text { Rileya sp.5 Eurytoma } \text { sp. } 7\end{array}$ & Myrtaceae & E. rotundifolia & cilíndrica caulinar & S.rotundifoliorum \\
\hline $\begin{array}{l}\text { Eurytomidae: } \\
\text { Eurytoma } \text { sp. } 8 \\
\text { Rileya } \text { sp. } 3,7\end{array}$ & Myrtaceae & E. uniflora & cônica foliar & C. profusa \\
\hline $\begin{array}{l}\text { Eurytomidae: } \\
\text { Rileya } \text { sp. } 3\end{array}$ & Myrtaceae & E. uniflora & $\begin{array}{l}\text { triangular foliar/ } \\
\text { fruto }\end{array}$ & Cecidomyiinae \\
\hline Eurytomidae & Myrtaceae & E. uniflora & circular foliar & N. eugeniae \\
\hline Eurytomidae & Myrtaceae & M. ovata & gema* & M. maricensis \\
\hline Eurytomidae & Myrtaceae & N. obscura & cônica foliar* & S. mina \\
\hline $\begin{array}{l}\text { Eurytomidae: } \\
\text { Rileya } \mathrm{sp} .3 \\
\text { Eurytoma } \mathrm{sp} .\end{array}$ & Nyctaginaceae & G. opposita & globosa foliar & B. robusta \\
\hline $\begin{array}{l}\text { Eurytomidae: } \\
\text { Rileya } \mathrm{sp} 3 \\
\text { Eurytoma } \text { sp.9 }\end{array}$ & Nyctaginaceae & G. opposita & circular foliar & B. elongata \\
\hline $\begin{array}{l}\text { Eurytomidae: } \\
\text { Eurytoma sp. } 9 \text { Rileya } \text { sp. } 3\end{array}$ & Nyctaginaceae & G. opposita & triangular foliar & B. acaudata \\
\hline Eurytomidae & Nyctaginaceae & G. opposita & foliar roxa* & Bruggmannia sp.1 \\
\hline $\begin{array}{l}\text { Eurytomidae: } \\
\text { Rileya sp.3, } 5 \text { e } 7\end{array}$ & Nyctaginaceae & G. opposita & espess.caulinar* & P. guapirae \\
\hline $\begin{array}{l}\text { Eurytomidae: } \\
\text { Rileya } \text { sp. } 7\end{array}$ & Rubiaceae & B. verticillata & inflorescência & A. borreriae \\
\hline
\end{tabular}


Tabela 1. Continuação...

\begin{tabular}{|c|c|c|c|c|}
\hline Famílias de Hymenoptera & $\begin{array}{c}\text { Famílias da planta } \\
\text { hospedeira }\end{array}$ & $\begin{array}{c}\text { Espécie da planta } \\
\text { hospedeira }\end{array}$ & Morfotipo da galha & $\begin{array}{c}\text { Cecidomyiidae } \\
\text { galhador }\end{array}$ \\
\hline $\begin{array}{l}\text { Eurytomidae: } \\
\text { Rileya } \mathrm{sp} .8\end{array}$ & Sapindaceae & P.weinmanniaefolia & cônica foliar & P. ampla \\
\hline $\begin{array}{l}\text { Eurytomidae: } \\
\text { Rileya sp. } 8\end{array}$ & Sapindaceae & P.weinmanniaefolia & espess. da gavinha & Cecidomyiinae \\
\hline Eurytomidae: Eurytoma sp. & Sapotaceae & M. subsericea & foliar circular* & Cecidomyiidi \\
\hline $\begin{array}{l}\text { Eurytomidae: } \\
\text { Eurytoma sp. } 10\end{array}$ & Sapotaceae & M. subsericea & globular foliar & Asphondyliini \\
\hline Eurytomidae & Sapotaceae & P. venosa & circular foliar & L. singularis \\
\hline Mymaridae & Erythroxylaceae & E. ovalifolium & cônica (gema) & Cecidomyiinae \\
\hline Mymaridae & Erythroxylaceae & E. ovalifolium & triangular foliar & Lasiopteridi \\
\hline Mymaridae & Euphorbiaceae & S. glandulosa & $\begin{array}{l}\text { enrolamento da } \\
\text { margem foliar }\end{array}$ & Cecidomyiidi \\
\hline Mymaridae & Malpighiaceae & B. sericea & circular foliar & Oligotrophini \\
\hline Mymaridae & Myrtaceae & E. copacabanensis & fusiforme foliar & S. teralobae \\
\hline Mymaridae & Myrtaceae & E. uniflora & cônica foliar & C. profusa \\
\hline Platygastridae: Synopeas sp. & Boraginaceae & C. verbenacea & globosa foliar & C. globosa \\
\hline Platygastridae & Boraginaceae & C. verbenacea & espess.nervura & Lopesiini \\
\hline $\begin{array}{l}\text { Platygastridae: } \\
\text { Platygaster sp. }\end{array}$ & Burseraceae & P. brasiliense & foliar & Cecidomyiinae \\
\hline $\begin{array}{l}\text { Platygastridae: } \\
\text { Platygaster sp. }\end{array}$ & Celastraceae & M. ovalifolia & foliar circular & M. distincta \\
\hline $\begin{array}{l}\text { Platygastridae: } \\
\text { Platygaster sp. }\end{array}$ & Clusiaceae & C. hilariana & circular foliar & C. granulosa \\
\hline $\begin{array}{l}\text { Platygastridae: } \\
\text { Inostemma } \mathrm{sp} . \\
\text { Platygaster sp. }\end{array}$ & Clusiaceae & C. lanceolata & esferóide foliar & C. nitida \\
\hline Platygastridae & Euphorbiaceae & S. glandulosa & esférica (gema)* & S. spherica \\
\hline Platygastridae & Euphorbiaceae & S. glandulosa & $\begin{array}{l}\text { enrolamento da } \\
\text { margem foliar }\end{array}$ & Cecidomyiidi \\
\hline $\begin{array}{l}\text { Platygastridae: } \\
\text { Platygaster sp. }\end{array}$ & Fabaceae & I. laurina & espess. nervura & Neolasioptera sp. \\
\hline $\begin{array}{l}\text { Platygastridae: } \\
\text { Platygaster sp. }\end{array}$ & Loranthaceae & S. maricensis & ovóide na nervura & A. maricensis \\
\hline Platygastridae & Malpighiaceae & B. sericea & espess.caulinar* & Cecidomyiinae \\
\hline Platygastridae: Synopeas sp. & Myrtaceae & E. multiflora & cilíndrica caulinar & Stephomyia sp. \\
\hline Platygastridae: Synopeas sp. & Myrtaceae & E.multiflora & claviforme & S. clavata \\
\hline Platygastridae & Myrtaceae & E. rotundifolia & cilíndrica caulinar* & S. rotundifoliorum \\
\hline $\begin{array}{l}\text { Platygastridae: } \\
\text { Platygaster sp. }\end{array}$ & Myrtaceae & E. rotundifolia & circular foliar & D. globosa \\
\hline Platygastridae & Myrtaceae & E. uniflora & cônica foliar* & C. profusa \\
\hline Platygastridae & Myrtaceae & E. uniflora & triangular foliar* & Cecidomyiinae \\
\hline Platygastridae & Myrtaceae & M. ovata & globular foliar* & Dasineura sp.1 \\
\hline Platygastridae & Myrtaceae & M. floribunda & $\begin{array}{l}\text { enrolamento da } \\
\text { margem foliar* }\end{array}$ & D. myrciariae \\
\hline Platygastridae & Myrtaceae & N. obscura & cônica foliar & S. mina \\
\hline $\begin{array}{l}\text { Platygastridae: } \\
\text { Platygaster sp. }\end{array}$ & Nyctaginaceae & G. opposita & globosa foliar & B. robusta \\
\hline $\begin{array}{l}\text { Platygastridae: } \\
\text { Platygaster sp. }\end{array}$ & Nyctaginaceae & G. opposita & circular foliar & B. elongata \\
\hline Platygastridae & Nyctaginaceae & G. opposita & espess.caulinar* & P. guapirae \\
\hline Platygastridae: & Ochnaceae & O. cuspidata & barril foliar* & Contarinia sp.1 \\
\hline
\end{tabular}


Tabela 1. Continuação...

\begin{tabular}{|c|c|c|c|c|}
\hline Famílias de Hymenoptera & $\begin{array}{c}\text { Famílias da planta } \\
\text { hospedeira }\end{array}$ & $\begin{array}{c}\text { Espécie da planta } \\
\text { hospedeira }\end{array}$ & Morfotipo da galha & $\begin{array}{c}\text { Cecidomyiidae } \\
\text { galhador }\end{array}$ \\
\hline $\begin{array}{l}\text { Platygastridae: } \\
\text { Platygaster sp. }\end{array}$ & Rubiaceae & $\begin{array}{l}\text { Diodia gymnocephala } \\
\text { (DC) K. Schum. }\end{array}$ & inflorescência & $\begin{array}{l}\text { Clinodiplosis diodiae } \\
\text { Maia, } 2001\end{array}$ \\
\hline Platygastridae: Leptacis sp. & Sapindaceae & P.weinmanniaefolia & cônica foliar & P. ampla \\
\hline $\begin{array}{l}\text { Platygastridae: } \\
\text { Platygaster sp. }\end{array}$ & Sapotaceae & M. subsericea & foliar circular & Cecidomyiidi \\
\hline Platygastridae & Sapotaceae & M. subsericea & tubular foliar & Asphondyliini \\
\hline Platygastridae & Sapotaceae & P. caimito & tubular foliar* & Y. pouteriae \\
\hline Platygastridae & Sapotaceae & P. venosa & circular foliar* & L. singularis \\
\hline $\begin{array}{l}\text { Platygastridae: } \\
\text { Platygaster sp. }\end{array}$ & Solanaceae & Solanum inaequale Vell. & esferóide foliar & Cecidomyiinae \\
\hline Platygastridae & Verbenaceae & L. camara & globosa foliar* & S. lantanae \\
\hline $\begin{array}{l}\text { Pteromalidae: } \\
\text { Lyrcus sp. }\end{array}$ & Boraginaceae & C. verbenacea & globosa foliar & C. globosa \\
\hline Pteromalidae & Boraginaceae & C. verbenacea & espess.nervura & Lopesiini \\
\hline Pteromalidae & Boraginaceae & C. verbenacea & inflorescência & Asphondylia sp.1 \\
\hline Pteromalidae & Celastraceae & M. ovalifolia & foliar circular & M. distincta \\
\hline Pteromalidae & Chrysobalanaceae & C. ovalifolia & $\begin{array}{l}\text { enrolamento da } \\
\text { margem foliar }\end{array}$ & L. marginalis \\
\hline Pteromalidae & Clusiaceae & C. lanceolata & esferóide foliar & C. nitida \\
\hline Pteromalidae & Erythroxylaceae & E.ovalifolium & botão floral & Asphondylia sp.1 \\
\hline Pteromalidae & Erythroxylaceae & E. ovalifolium & cônica (gema) & Cecidomyiinae \\
\hline Pteromalidae & Erythroxylaceae & E.ovalifolium & triangular foliar & Lasiopteridi \\
\hline Pteromalidae & Euphorbiaceae & S. glandulosa & esférica (gema) & S. spherica \\
\hline Pteromalidae & Loranthaceae & P. dichrous & circular foliar & C. maricaensis \\
\hline Pteromalidae & Malpighiaceae & B. sericea & espess. caulinar & Cecidomyiinae \\
\hline Pteromalidae & Myrsinaceae & R. parvifolia & circular foliar & Cecidomyiinae \\
\hline Pteromalidae & Myrtaceae & M. floribunda & $\begin{array}{l}\text { enrolamento da } \\
\text { margem foliar }\end{array}$ & D. myrciariae \\
\hline Pteromalidae & Myrtaceae & M. floribunda & bivalva (gema) & M. bivalva \\
\hline Pteromalidae & Myrtaceae & N. obscura & imbricada (gema) & $N$. robusta \\
\hline Pteromalidae & Myrtaceae & N. obscura & tubular foliar & Cecidomyiinae \\
\hline Pteromalidae & Verbenaceae & L. camara & globosa foliar & S. lantanae \\
\hline Scelionidae & Verbenaceae & L. camara & globosa foliar & S. lantanae \\
\hline $\begin{array}{l}\text { Signiphoridae: } \\
\text { Signiphora sp. }\end{array}$ & Fabaceae & I. laurina & fruto & Cecidomyiinae \\
\hline Tanaostigmatidae & Fabaceae & I. laurina & tubular foliar & Cecidomyiinae \\
\hline Torymidae & Asclepiadaceae & P. asteria & botão floral & A. peploniae \\
\hline $\begin{array}{l}\text { Torymidae: Dimeromicrus } \\
\text { cecidomyiae }\end{array}$ & Asteraceae & M. hoehnei & espess. caulinar & Alycaulus sp. \\
\hline $\begin{array}{l}\text { Torymidae: } \\
\text { D. cecidomyiae } \\
\text { Torymoides sp. Torymus sp. }\end{array}$ & Boraginaceae & C. verbenacea & globosa foliar & C. globosa \\
\hline $\begin{array}{l}\text { Torymidae: } \\
\text { D. cecidomyiae }\end{array}$ & Boraginaceae & C. verbenacea & espess.nervura & Lopesiini \\
\hline $\begin{array}{l}\text { Torymidae: } \\
\text { D. cecidomyiae }\end{array}$ & Boraginaceae & C. verbenacea & inflorescência & Asphondylia sp.1 \\
\hline $\begin{array}{l}\text { Torymidae: } \\
\text { D. cecidomyiae }\end{array}$ & Celastraceae & M. ovalifolia & foliar circular* & M. distincta \\
\hline Torymidae & Chrysobalanaceae & C. ovalifolia & foliar circular* & D. couepiae \\
\hline Torymidae & & & $\begin{array}{l}\text { enrolamento da } \\
\text { margem foliar }\end{array}$ & L. marginalis \\
\hline
\end{tabular}


Tabela 1. Continuação...

\begin{tabular}{|c|c|c|c|c|}
\hline Famílias de Hymenoptera & $\begin{array}{c}\text { Famílias da planta } \\
\text { hospedeira }\end{array}$ & $\begin{array}{c}\text { Espécie da planta } \\
\text { hospedeira }\end{array}$ & Morfotipo da galha & $\begin{array}{c}\text { Cecidomyiidae } \\
\text { galhador }\end{array}$ \\
\hline $\begin{array}{l}\text { Torymidae: } \\
\text { D. cecidomyiae }\end{array}$ & Convolvulaceae & $\begin{array}{l}\text { Jacquemontia } \\
\text { holosericea (Weinman) } \\
\text { O'Donell }\end{array}$ & botão floral & Schizomyia sp. \\
\hline $\begin{array}{l}\text { Torymidae: } \\
\text { D. cecidomyiae }\end{array}$ & Erythroxylaceae & E. ovalifolium & botão floral* & Asphondylia sp.1 \\
\hline Torymidae & Erythroxylaceae & E. ovalifolium & triangular foliar* & Lasiopteridi \\
\hline $\begin{array}{l}\text { Torymidae: } \\
\text { D. cecidomyiae }\end{array}$ & Euphorbiaceae & S. glandulosa & esférica (gema)* & S. spherica \\
\hline Torymidae & Fabaceae & $\begin{array}{l}\text { Dalbergia ecastophylla } \\
\text { L. Taub. }\end{array}$ & discóide foliar & $\begin{array}{l}\text { Lopesia grandis } \\
\text { Maia, } 2001\end{array}$ \\
\hline $\begin{array}{l}\text { Torymidae: } \\
\text { D. cecidomyiae }\end{array}$ & Fabaceae & I. maritima & espess.nervura* & Neolasioptera sp.1 \\
\hline Torymidae & Fabaceae & $\begin{array}{l}\text { Stylosanthes guianensis } \\
\text { Sw. }\end{array}$ & inflorescência & Lestodiplosis sp. \\
\hline Torymidae & Malpighiaceae & B. sericea & botão floral* & B. byrsonimae \\
\hline $\begin{array}{l}\text { Torymidae: } \\
\text { D. cecidomyiae }\end{array}$ & Myrtaceae & E. rotundifolia & cilíndrica caulinar* & S. rotundifoliorum \\
\hline Torymidae & Myrtaceae & E. rotundifolia & circular foliar* & D. globosa \\
\hline Torymidae & Myrtaceae & E. rotundifolia & $\begin{array}{l}\text { enrolamento da } \\
\text { margem foliar }\end{array}$ & Cecidomyiidi \\
\hline Torymidae & Myrtaceae & E. uniflora & cônica foliar* & C. profusa \\
\hline $\begin{array}{l}\text { Torymidae: } \\
\text { D. cecidomyiae }\end{array}$ & Myrtaceae & M. floribunda & $\begin{array}{l}\text { enrolamento da } \\
\text { margem foliar* }\end{array}$ & D. myrciariae \\
\hline $\begin{array}{l}\text { Torymidae: } \\
\text { D. cecidomyiae }\end{array}$ & Myrtaceae & N. obscura & triangular foliar & Cecidomyiinae \\
\hline Torymidae & Nyctaginaceae & G. opposita & triangular foliar & B. acaudata \\
\hline Torymidae & Rubiaceae & D. gymnocephala & inflorescência & C. diodiae \\
\hline $\begin{array}{l}\text { Torymidae: } \\
\text { D. cecidomyiae }\end{array}$ & Sapindaceae & P.weinmanniaefolia & cônica foliar & P. ampla \\
\hline $\begin{array}{l}\text { Torymidae: } \\
\text { D. cecidomyiae }\end{array}$ & Sapotaceae & M. subsericea & foliar circular* & Cecidomyiidi \\
\hline Torymidae & Sapotaceae & M. subsericea & gema & Cecidomyiidi \\
\hline $\begin{array}{l}\text { Torymidae: } \\
\text { D. cecidomyiae }\end{array}$ & Sapotaceae & P. caimito & tubular foliar & Y. pouteriae \\
\hline Torymidae & Sapotaceae & P. venosa & esferóide foliar & L. singularis \\
\hline $\begin{array}{l}\text { Torymidae: } \\
\text { D. cecidomyiae }\end{array}$ & Verbenaceae & L. camara & globosa foliar* & S. lantanae \\
\hline
\end{tabular}

Os micro-himenópteros estudados estão associados com 26 gêneros de Cecidomyiidae, com destaque para Asphondylia Loew, 1850; Bruggmannia Tavares, 1906, Clinodiplosis Kieffer, 1894; Dasineura Rondani, 1840; Lopesia Rübsaamen 1908, Neolasioptera Felt, 1908 e Stephomyia Tavares, 1916 pelo maior número de registros verificados. Esses gêneros diferem muito em número de espécies conhecidas e distribuição geográfica. Asphondylia e Dasineura são cosmopolitas e incluem, respectivamente, 271 e 448 espécies no mundo; Clinodiplosis também cosmopolita inclui um número menor de espécies (93); Lopesia ocorre nas regiões neártica, neotropical e afro-tropical e possui apenas 13 espécies descritas; Neolasioptera tem distribuição mais restrita, ocorrendo nas regiões neártica e neotropical e inclui um grande número de espécies (133), já Bruggmannia e Stephomyia são gêneros pouco diversificados, com 18 e sete espécies conhecidas e exclusivamente neotropicais (Gagné, 2004). Esses dados mostram que a incidência de micro-himenópteros não está necessariamente relacionada nem com a diversidade dos gêneros galhadores e nem com maiores áreas de ocorrência dos mesmos.

Não foi observada nenhuma relação específica entre as seguintes famílias de parasitóides - Aphelinidae, Bethylidae, Braconidae, Encyrtidae, Eulophidae, Eupelmidae, Eurytomidae, Mymaridae, Platygastridae, Pteromalidae e Torymidae - e os gêneros galhadores. Diferentemente, os Elasmidae, Scelionidae e Signophoridae ocorreram, cada qual, em associação com um único gênero galhador. Especificidade também foi verificada em relação aos Tanaostigmatidae, inquilinos de um único morfotipo de galhas.

Os micro-himenópteros foram obtidos de galhas que se desenvolveram em órgãos vegetativos - folha (no limbo, parênquima, nervura, margem foliar e pecíolo); caule e gema (apical e lateral) - e em órgãos reprodutivos (botão floral, pedúnculo floral, inflorescência 
e fruto), sendo mais freqüentemente obtidos de galhas foliares. Como $60 \%$ das galhas das Restingas do Estado do Rio desenvolvem-se em folhas, o resultado encontrado não indica preferência por um órgão vegetal particular, e sugere que a maior freqüência de galhas nas folhas é responsável pela maior freqüência dos micro-himenópteros nesse órgão.

A seguir, são apresentadas informações, em ordem alfabética, sobre as famílias de micro-himenópteros obtidas a partir de galhas de Cecidomyiidae em Restingas do Estado do Rio de Janeiro.

\section{Aphelinidae}

Parasitóides de uma grande variedade de insetos. São conhecidas mil espécies no mundo em 38 gêneros. No Brasil, ocorrem 16 espécies em dois gêneros (Perioto \& Tavares 1999). Nas Restingas fluminenses, os Aphelinidae estão associados com cinco espécies de plantas (cada qual representando um gênero e família distintos) e cinco morfotipos de galhas. Foram encontrados quase que exclusivamente em galhas foliares, com exceção de um registro em galhas de botão floral. Atuaram como parasitóides de três gêneros de Cecidomyiidae galhadores: Asphondylia Loew, 1850, Dasineura Rondani, 1840 e Clusiamyia Maia, 1997 (Tabela 1).

\section{Bethylidae}

Parasitóides de larvas e ocasionalmente de pupas de Coleoptera e Lepidoptera (Naumann 1991). No Brasil, ocorrem 112 espécies em 16 gêneros (De Santis 1980). Nas Restingas fluminenses, foram encontrados como parasitóides em enrolamentos da margem da folha, induzidos por Lopesia sp. e Dasineura myrciariae, em Protium brasiliense (Spr.) Engl. (Burseraceae) e Myrciaria floribunda, respectivamente. O parasitóide associado com Lopesia sp. foi identificado em nível genérico como Goniozus Förster, 1856 (ocorrência assinalada por Maia et al., 2002). O registro em Myrciaria floribunda é novo (Tabela 1).

\section{Braconidae}

Parasitóides de insetos, comuns em todo o mundo, porém mais diversificados nas áreas mais quentes. No Brasil, ocorrem 475 espécies em 99 gêneros (De Santis 1980). Nas Restingas fluminenses, estão associados com sete espécies de plantas (cinco gêneros e quatro famílias). Foram encontrados apenas em galhas foliares induzidas por quatro gêneros distintos de Cecidomyiidae: Dasineura Felt, 1908, Neolasioptera Felt, 1908, Stephomyia Tavares, 1916 e Paulliniamyia Maia, 2001. São assinalados, pela primeira vez, em cinco espécies de plantas (Tabela 1).

\section{Elasmidae}

Ectoparasitóides de insetos, especialmente de lagartas de Lepidoptera. Família com um único gênero, Elasmus Westwood, 1883 com 200 espécies. No Brasil, ocorrem cinco espécies (Perioto \& Tavares 1999). Nas Restingas fluminenses, uma única espécie é encontrada, tendo sido assinalada por Maia et al. (2002) em galhas de Neolasioptera sp. em Inga laurina (Sw.) Willd (Fabaceae) (Tabela 1).

\section{Eulophidae}

Ecto ou endoparasitóides de ovos, larvas e pupas de vários insetos, incluindo Diptera (particularmente Cecidomyiidae), Hymenoptera (Cynipoidea), Coleoptera e ácaros Eriophydae (La Salle 1994). Compreendem 3.300 espécies em 280 gêneros. No Brasil, ocorrem 103 espécies em 48 gêneros (Perioto \& Tavares 1999). Nas Restingas fluminenses, estão associados a 34 espécies de plantas (28 gêneros e 22 famílias), ocorrendo em 58 morfotipos de galhas, principal- mente em folhas. Estão representados por 12 gêneros: Aprostocetus Westwood, 1833; Chrysonotomyia Ashmead, 1904; Cirrospilus Westwood, 1832; Closterocerus Westwood, 1833; Galeopsomyia Girault, 1916; Hadrotrichodes LaSalle, 1994 ; Horismenus Walker, 1843; Miotropis Thomson, 1875; Pentastichus Ashmead, 1894; Proacrias Ihering, 1914; Quadrastichus Girault, 1913; Xanthellum Erdös \& Novicky, 1951 e sete gêneros novos. Trata-se do primeiro registro de Quadrastichus para o Brasil. Os Eulophidae foram encontrados em galhas induzidas por 22 gêneros distintos de Cecidomyiidae e são assinalados, pela primeira vez, em 29 morfotipos de galhas (Tabela 1).

\section{Encyrtidae}

Maioria parasitóide de Coccoidea (Hemiptera). No mundo, são conhecidas 3.200 espécies em 450 gêneros. No Brasil, ocorrem 96 espécies em 62 gêneros (Perioto \& Tavares 1999). Nas Restingas fluminenses, estão associados a seis espécies de planta (seis gêneros e seis famílias) e sete morfotipos de galhas que se desenvolvem em diferentes órgãos da planta (folha, botão floral, gema e caule), induzidos por cinco gêneros distintos de Cecidomyiidae: Asphondylia, Bruggmanniella Tavares, 1909, Dasineura, Myrciariamyia Maia,1995 e Neolasioptera Felt, 1908 (Tabela 1).

\section{Eupelmidae}

Maioria parasitóide ou hiperparasitóide facultativo das principais ordens de insetos. Compreendem 850 espécies em 45 gêneros. No Brasil, ocorrem 27 espécies em 12 gêneros (Perioto \& Tavares 1999). Nas Restingas fluminenses, estão associados com 28 espécies de plantas (21 gêneros e 17 famílias) e 42 morfotipos de galhas (Tabela 1). Ocorreram em vários órgãos vegetais, sendo mais frequientes nas folhas. São assinalados, pela primeira vez, em 33 morfotipos de galhas e estão associados com 16 gêneros distintos de Cecidomyiidae galhadores.

\section{Eurytomidae}

Maioria parasitóide, algumas espécies são fitófagas. No mundo, são conhecidas 1100 espécies em 70 gêneros e no Brasil 59 espécies em 22 gêneros (Perioto \& Tavares 1999). Nas Restingas fluminenses, estão associados com 22 espécies de plantas (18 gêneros e 15 famílias) e 33 morfotipos de galhas, induzidos por 16 gêneros distintos de Cecidomyiidae. Ocorreram em vários órgãos vegetais (folha, botão floral, fruto, caule, gavinha, gema), predominando nas folhas e são assinalados, pela primeira vez, em sete morfotipos de galhas. Estão representados por três gêneros: Eurytoma Illiger, 1807; Rileya Ashmead, 1888 e Sycophila Walker, 1871 (Tabela 1).

\section{Mymaridae}

Parasitóides de ovos, principalmente de Hemiptera, Psocoptera, Coleoptera, Diptera e Orthoptera. Compreendem 1.300 espécies em 100 gêneros. No Brasil, tem-se registro de 21 espécies em nove gêneros (Perioto \& Tavares 1999). Nas Restingas fluminenses, estão associados com cinco espécies de planta (quatro gêneros e quatro famílias) e seis morfotipos de galhas (5 foliares). Todos os registros são novos (Tabela 1).

\section{Platygastridae}

Parasitóides de insetos, principalmente de Cecidomyiidae galhadores. No Brasil, ocorrem 15 espécies em 5 gêneros (De Santis 1980). Nas Restingas fluminenses, estão associados com 24 espécies de plantas (em 20 gêneros e 16 famílias) e 32 morfotipos de galhas (Tabela), estando representados por quatro gêneros: Inostemma Haliday, 1833 ; Leptacis Foerster, 1856; Platygaster Latreille, 1809 
e Synopeas Foerster, 1856. São assinalados, pela primeira vez, em 12 morfotipos de galhas (Tabela 1) e estão associados a 16 gêneros distintos de Cecidomyiidae. Ocorreram com maior freqüência em folhas, mas também em galhas que se desenvolveram no caule, gema e inflorescência.

\section{Pteromalidae}

Maioria parasitóide e alguns predadores de insetos. No mundo são conhecidas 3.000 espécies em 551 gêneros. No Brasil, ocorrem 110 espécies em 55 gêneros (Perioto \& Tavares 1999). Nas Restingas fluminenses, estão associados com doze espécies de plantas (doze gêneros e onze famílias), 18 morfotipos de galhas (a maioria foliar) e 11 gêneros de Cecidomyiidae (Tabela 1). Todos os registros são novos, exceto em Cordia verbenacea DC. (Boraginaceae).

\section{Scelionidae}

Parasitóides de ovos de insetos e aranhas. No Brasil, ocorrem 68 espécies em 10 gêneros (De Santis 1980). Nas Restingas fluminenses, uma única espécie foi encontrada em associação com galhas foliares esféricas e pilosas induzidas por Schismatodiplosis lantanae Rübsaamen, 1907 (Cecidomyiidae) em Lantana camara L. (Verbenaceae). Novo registro.

\section{Signiphoridae}

Parasitóides de Coccoidea, Aleyrodidae, Psyllidae (Hemiptera) e Diptera (Woolley 1988). No mundo, são conhecidos seis ou sete gêneros com poucas espécies (Rao \& Hayat, 1985). No Brasil, ocorrem 15 espécies de um único gênero. Nas Restingas fluminenses, Signiphora sp. foi encontrada como parasitóide de larvas de Cecidomyiidae em frutos de Inga laurina (Fabaceae), tendo sido registrada por Maia et al. (2002).

\section{Tanaostigmatidae}

Galhadores e inquilinos de galhas (Naumann 1991). Família com 50 espécies em 15 gêneros (Perioto \& Tavares 1999). A maioria é conhecida da região neotropical e Australásia (Rao \& Hayat 1985). No Brasil, ocorrem 17 espécies em quatro gêneros (Perioto \& Tavares, 1999). Nas Restingas fluminenses, uma única espécie foi encontrada como inquilina em galhas tubulares foliares induzidas por Cecidomyiidae em Inga laurina (Fabaceae), tendo sido registrada por Maia et al., 2002.

\section{Torymidae}

Maioria parasitóide de Cynipidae, Eurytomidae e Cecidomyiidae galhadores; algumas espécies inquilinas de galhas. No mundo, são conhecidas 900 espécies em 65 gêneros. No Brasil, ocorrem 26 espécies em seis gêneros (Perioto \& Tavares 1999). Nas Restingas fluminenses, estão associados com 23 espécies de plantas (21 gêneros e 16 famílias), 30 morfotipos de galhas (principalmente foliares) e 16 gêneros de Cecidomyiidae. Estão representados por três gêneros: Dimeromicrus Crawford, 1910; Torymoides Walker, 1871 e Torymus Dalman, 1820. Vinte e três registros são novos.

\section{Conclusões}

Nas Restingas do Estado do Rio de Janeiro, a frequiência registrada de micro-himenópteros associados a galhas de Cecidomyiidae é muito alta (95\%), superando significativamente os valores encontrados para Restingas paulistas e para o cerrado mineiro.

Os micro-himenópteros associados a galhas compreendem representantes de 15 famílias distintas. Entre elas, os Eulophidae, Eupelmidae e Platygastridae mostraram maior diversidade de hospedeiros.
Os micro-himenópteros foram obtidos de 45 espécies de plantas hospedeiras (35 gêneros e 25 famílias), sendo que as Myrtaceae, Fabaceae e Malpighiaceae destacaram-se pela maior riqueza de famílias de parasitóides associados.

A diversidade dos parasitóides associados às galhas de Cecidomyiidae parece não estar relacionada nem com a riqueza de espécies da família de planta hospedeira e nem com a riqueza de galhas.

Os micro-himenópteros ocorreram em galhas desenvolvidas em órgãos vegetativos e reprodutivos, predominando nas galhas foliares.

Eles estão associados com 26 gêneros de Cecidomyiidae, com destaque para Asphondylia Loew, 1850; Bruggmannia Tavares, 1906, Clinodiplosis Kieffer, 1894; Dasineura Rondani, 1840; Lopesia Rübsaamen 1908, Neolasioptera Felt, 1908 e Stephomyia Tavares, 1916.

A riqueza das famílias dos micro-himenópteros associados às galhas não está necessariamente relacionada à riqueza dos gêneros galhadores.

Não foi observada nenhuma relação específica entre as seguintes famílias de parasitóides - Aphelinidae, Bethylidae, Braconidae, Encyrtidae, Eulophidae, Eupelmidae, Eurytomidae, Mymaridae, Platygastridae, Pteromalidae e Torymidae - e os gêneros galhadores.

Apenas quatro famílias de micro-himenópteros mostraram especificidade em relação ao hospedeiro: Elasmidae, Scelionidae, Signophoridae e Tanaostigmatidae.

Cerca de 135 novos registros de plantas hospedeiras e/ou de morfotipos de galhas foram assinalados, o que evidencia a falta de informações sobre esses micro-himenópteros em Restingas.

A alta incidência de micro-himenópteros associada à grande diversidade de plantas hospedeiras e de espécies galhadoras corrobora a importância ecológica desses parasitóides como inimigos naturais dos Cecidomyiidae e reforça a possibilidade de serem utilizados como agentes de controle biológico.

\section{Agradecimentos}

Aos Drs. Ruy Alves e Andréa Costa (Museu Nacional/UFRJ) pela identificação das plantas hospedeiras, à FAPERJ (Proc. 171.290/06) e ao CNPq (Proc. 472084/2007-0) pelo suporte financeiro.

\section{Referências Bibliográficas}

ARAÚJO, D.S.D. \& HENRIQUES, P.B. 1984. Análise florística das restingas do Estado do Rio de Janeiro. In Restingas: origem, estrutura e processos (L.D. Lacerda, D.S.D. Araújo, R. Cerqueira \& B. Turq, orgs). CEUFF, Niterói, p. 159-193.

De SANTIS, L. 1980. Catálogo de los Himenópteros Brasileños de la serie parasítica: incluyendo Bethyoidea. Universidade Federal do Paraná, Curitiba.

FERNANDES, G.W. \& PRICE, P.W. 1992. The adaptative significance of insect gall distribution: survivorship of species in xeric and mesic habitats. Oecologia, 90:14-20.

FERRAZ, F.F.F. \& MONTEIRO, R.F. 2003. Complex interactions envolving a gall midge Myrciariamyia maricaensis Maia (Diptera, Cecidomyiidae), phytophagous modifiers and parasitoids. Rev. Bras. Zool. 20(3):433-437.

GAGNÉ, R.J. 1989. The Plant-Feeding gall midges of North America. Cornell University Press, Ithaca.

GAGNÉ, R.J. 2004. A catalog of the Cecidomyiidae (Diptera) of the world. Mem. Entomol. Soc. Wash. 25:408.

JOLY, A.B. 1977. Botânica: Introdução à taxonomia vegetal. 4 ed. Editora Nacional, São Paulo. 777 p. 
LASALLE, J. 1994. North America genera of Tetrastichinae (Hymenoptera: Eulophidae). J. Nat. Hist. 28(1):109-236.

MAIA, V.C. 1999. Artrópodes associados às galhas de Cecidomyiidae (Diptera) em Eugenia rotundifolia (Myrtaceae) e Clusia lanceolata (Clusiaceae) em uma restinga do Rio de Janeiro, Brasil. Iheringia, Zool. 87:75-79.

MAIA, V.C. \& De AZEVEDO, M.A.P. 2001a. Platygastridae (Hym., Scelionoidea) associated with Cecidomyiidae (Dipt.) galls at two restingas (coastal shrub zones) of Rio de Janeiro State (Brazil). Entomol. Mon. Mag. 137:149-153.

MAIA, V.C. \& De AZEVEDO, M.A.P. 2001b. Eurytomidae (Hym., Chalcidoidea) associated with Cecidomyiidae (Dipt.) galls in Rio de Janeiro State (Brazil). Entomol. Mon. Mag. 137:219-225.

MAIA, V.C. \& MONTEIRO, R.F. 1999. Espécies cecidógenas (Diptera, Cecidomyiidae) e parasitóides (Hymenoptera) associadas a Guapira opposita (Vell.) Reitz. (Nyctaginaceae) na restinga da Barra de Maricá, Rio de Janeiro. Rev. Bras. Zool. 16(2):483-487.

MAIA, V.C. \& TAVARES, M.T. 2000. Cordiamyia globosa Maia (Diptera, Cecidomyiidae): flutuação populacional e parasitóides (Hymenoptera) associados. Rev. Bras. Zool. 17(3):589-593.

MAIA, V.C., De AZEVEDO, M.A.P. \& COURI, M.S. 2002. New contribution to the knowledge of the gall midges (Diptera, Cecidomyiidae) from the restinga of Barra de Maricá (Rio de Janeiro, Brazil). Stud. Dipterol. $9(2): 447-452$
MONTEIRO, R.F., ODA, R.A.M., NARAHARA, K.L. \& CONSTANTINO, P.A.L. 2004. Galhas: Diversidade, Especificidade e Distribuição. In Pesquisa de Longa Duração na Restinga de Jurubatiba: Ecologia, História Natural e Conservação (C.F.D. da Rocha, F.A. Esteves \& F.R. Scarano, orgs). RiMa Editora, São Carlos, p. 127-141.

NAUMANN, I.D. 1991. Hymenoptera: 916-1000. In The Insects of Australia (CSIRO, orgs). 2 ed. Cornell University, Ithaca, p. 543-1137.

PERIOTO, N.W. \& TAVARES, M.T. 1999. Hymenoptera. In Biodiversidade do Estado de São Paulo, Brasil. 5. Invertebrados Terrestres (C.R.F. Brandão \& E.M. Cancello, eds). Museu de Zoóloga da Universidade de São Paulo, São Paulo, p. 156-168.

PRICE, P.W. 1984. Insect Ecology. 2 ed. Wiley-Interscience Publication, New York.

RAO, B.R.S. \& HAYAT, M. 1985. The Chalcidoidea (Insecta, Hymenoptera) of India and the adjacent countries. Part 1: reviews of families and keys to families and genera. Orient. Insects 19:163-310.

WOOLLEY, B. 1988. Philogeny and classification of the Signiphoridae (Hymenoptera, Chalcidoidea). Syst. Entomol. 139(4):465-501.

Recebido em 25/11/2008

Versão reformulada recebida em 25/05/2009

Publicado em 31/05/2009 